\title{
On the Derivation and Accuracy of Certain Formulas for Sample Sizes and Operating Characteristics of Nonsequential Sampling Procedures
}

\author{
Uttam Chand ${ }^{2}$
}

\begin{abstract}
Formulas are given that are needed for the computation of number of observations and operating characteristics of single sample acceptance sampling procedures based on tests of statistical hypotheses. Some of the same results may be obtained by reference to existing tables and curves located in widely scattered places. The hypotheses considered concern the means and standard deviations of certain populations (that is, binomial, Poisson, normal) where the test is made against a one-sided alternative. The comparison of two means or two variances as well as the test of a single mean or variance is discussed. The accuracy of the formulas is considered, and where approximations are involved, the results are compared with existing tables.
\end{abstract}

This paper develops and considers the question of the accuracy of certain formulas needed for the number of observations and operating characteristics of single-sample acceptance-sampling procedures based on statistical tests of hypotheses. For purposes of completeness the paper also contains formulas that are already known.

\section{Introduction}

It is now common knowledge among users of modern statistical tools that the characteristics of a sampling plan must be specified in terms of the risks of rejecting good material (Type I error: producer's risk) and accepting poor material (Type II error: consumer's risk). The problem of determining a sample size for a given probability of Type I error, which will minimize the probability of Type II error, has been considered by several authors, in particular [1 to 4]. ${ }^{3}$ This problem belongs to the category of that broad class of problems in the field of planned experimentation in which one is asked to provide adequate replication to detect treatment differences with desired amount of protection against taking wrong decisions. If for a certain sample size it is impossible to reduce simultaneously to small proportions the risks of two kinds it will be helpful to know this in advance.

Answers to most of the questions raised in this paper can be obtained from the existing published tables and curves. The effectiveness of these formulas in relation to assumptions and approximations that have been made in their derivation also has a theoretical interest. We shall restrict ourselves to the consideration of certain parametric hypotheses concerning means and standard deviations of certain populations, mainly against one-sided alternatives.

\section{Preliminaries and Notation}

In the ensuing sections $H_{1}$ denotes the null hypothesis, $H_{2}$ any one of a set of alternative hypo-

Revision of a paper written during the summer of 1947 when the author was a guest worker at the National Bureau of Standards. The manuscript was actually revised while the author was teaching at Boston University.

2 Present address, c/o P. V. Sukhatme, Indian Council of Agricultural Re search, New Delhi, India.

${ }^{3}$ Figures in brackets indicate the literature references at the end of this paper. theses, $\alpha$ the probability of rejecting the null hypothesis $H_{1}$ when true, and $\beta$ the probability of accepting $H_{1}$ when some alternative hypothesis, $H_{2}$, is true. In connection with the hypotheses concerning the means of certain populations in which the standard deviations are functionally related to the means and consequently unspecified, the reader will at once recognize that the acceptance-rejection criterion $A$ used for a statistic $T$ is not the best in the sense of the likelihood ratio test [5]. This difficulty, however, can be avoided by transformation of the original variables and has been indicated in the appropriate sections. In connection with the twosample problem the formulas assume equal sample sizes. These formulas can obviously be extended to cases in which it is desired to take unequal sample sizes of $N_{1}$ and $N_{2}$ that are assumed in advance to be functionally related.

\section{A General Formula Concerning Sample Size and Region of Rejection}

Let $x=x(N)$ be a normally distributed variable with mean $\mu_{1}$ and standard deviation (sd) $\sigma_{1}=$ $f\left(\mu_{1}\right) F(N)$ under $H_{1}$ and with mean $\mu_{2}$ and sd $\sigma_{2}=$ $f\left(\mu_{2}\right) F(N)$ under $H_{2}\left(\mu_{2}>\mu_{1}\right)$, where $F(N)$ is a certain function of the sample size $N$ and is independent of $\sigma$. We assume $x>A$ as the critical region and obtain

and

$$
\frac{1}{\sqrt{2 \pi}} \int_{K_{\alpha}}^{\infty} e^{-t^{2} / 2} d t=\alpha
$$

$$
\frac{1}{\sqrt{2 \pi}} \int_{K_{1-\beta}}^{\infty} e^{-t^{2} / 2} d t=1-\beta
$$

where $K_{\epsilon}$ is the standardized normal deviate exceeded with probability $\epsilon$ and actually

$$
\begin{gathered}
\frac{A-\mu_{1}}{f\left(\mu_{2}\right) F(N)}=K_{\alpha} \\
\frac{A-\mu_{2}}{f\left(\mu_{2}\right) F(N)}=K_{1-\beta}=-K_{\beta}
\end{gathered}
$$

Solving (1) and (2) for $F(N)$ and $A$ we obtain 


$$
\begin{aligned}
& F(N)=\frac{\mu_{2}-\mu_{1}}{K_{\alpha} f\left(\mu_{1}\right)+K_{\beta} f\left(\mu_{2}\right)} \\
& A=\frac{K_{\alpha} \mu_{2} f\left(\mu_{1}\right)+K_{\beta} \mu_{1} f\left(\mu_{2}\right)}{K_{\alpha} f\left(\mu_{1}\right)+K_{\beta} f\left(\mu_{2}\right)},
\end{aligned}
$$

which may also be written as

$$
\begin{gathered}
1=\frac{\mu_{2}-\mu_{1}}{K_{\alpha} \sigma_{1}+K_{\beta} \sigma_{2}} \\
A=\frac{K_{\alpha} \mu_{2} \sigma_{1}+K_{\beta} \mu_{1} \sigma_{2}}{K_{\alpha} \sigma_{1}+K_{\beta} \sigma_{2}},
\end{gathered}
$$

for later convenience in certain simplifications. We note in passing that in particular cases of application $f(\mu)$ will either be a function of $\mu$ (cf. binomial and Poisson), or it will not depend on $\mu$, in which case it will be a certain function of the population sd $\sigma$ (cf. normal) or a pure number (cf. transformed binomial and Poisson).

\section{Tests Relating to the Parameter of the Binomial Distribution}

\subsection{Single Binomial}

Consider a random sample of $N$ items drawn from an infinite population in which a proportion $P$ of the items possesses a definite attribute $B$ and let $p$ be the fraction of the items possessing $B$ in the sample. Then $E(p)=P$ and $\sigma(p)=\sqrt{P(1-P)} / \sqrt{N}$. Our hypotheses are $H_{1}: P=P_{1}$ and $H_{2}: P=P_{2}\left(P_{2}>P_{1}\right)$. Using the normal approximation for the binomial variable $p,(3)$ and (4) imply

$$
\begin{aligned}
& N \cong\left(\frac{K_{\beta} \sqrt{P_{2}\left(1-P_{2}\right)}+K_{\alpha} \sqrt{P_{1}\left(1-P_{1}\right)}}{P_{2}-P_{1}}\right)^{2} \\
& A \cong \frac{K_{\beta} P_{1} \sqrt{P_{2}\left(1-P_{2}\right)}+K_{\alpha} P_{2} \sqrt{P_{1}\left(1-P_{1}\right)}}{K_{\alpha} \sqrt{P_{1}\left(1-P_{1}\right)}+K_{\beta} \sqrt{P_{2}\left(1-P_{2}\right)}}
\end{aligned}
$$

While (7) determines directly the sample size that will (approximately) guarantee a specified $\alpha$ and $\beta$, this may also be looked upon as providing the values of the probability of accepting $H_{1}$ for different values of $P$ for given $\quad ⿱ 乛$ and $\alpha$. For example (7) yields

$$
K_{\beta} \cong \frac{\sqrt{N}\left(P_{2}-P_{1}\right)-K_{\alpha} \sqrt{P_{1}\left(1-P_{1}\right)}}{\sqrt{P_{2}\left(1-P_{2}\right)}} .
$$

The inverse sine transformation $\theta=2 \quad \sin ^{-1} \sqrt{p}$ where $\theta$ is measured in radians $\lceil 16\rceil$ avoids the difficulty of the dependence of the standard deviation of of $p$ on unknown $P$, since $\theta$ is approximately normally distributed about $2 \sin ^{-1} \sqrt{P}$ with sd $\sigma(\theta) \cong \sqrt{(1 / N)}$. In terms of the transformed quantities we obtain

$$
\begin{aligned}
& N \cong \frac{1}{4}\left(\frac{K_{\alpha}+K_{\beta}}{\sin ^{-1} \sqrt{P_{2}}-\sin ^{-1} \sqrt{P_{1}}}\right)^{2} \\
& A \cong 2\left(\frac{K_{\beta} \sin ^{-1} \sqrt{P_{1}}+K_{\alpha} \sin ^{-1} \sqrt{P_{2}}}{K_{\alpha}+K_{\beta}}\right)
\end{aligned}
$$

$$
K_{\beta} \cong 2 \sqrt{\bar{N}}\left(\sin ^{-1} \sqrt{P_{2}}-\sin ^{-1} \sqrt{P_{1}}\right)-K_{\alpha} .
$$

We have derived (7) and (10) to illustrate the use of the results given in section 3 . The comparison of the two formulas raises questions of quite a complicated nature. We have so far not found any convenient yardstick with which to compare their accuracy. In the light of the fact that the critical region $\theta>A$ (11) has certain theoretical advantages against $p>A$ (8) there is the temptation to recommend (10). As the following example will indicate ( $\alpha=.05, P_{1}=0.1$ and $P_{2}=0.2$ ) the recommendation has nothing to do with the relative magnitude of the values of $N$ given by (7) and (10).

\begin{tabular}{|c|c|c|c|c|}
\hline$\beta=$ & 0.20 & 0.10 & 0.05 & 0.01 \\
\hline \multicolumn{1}{c|}{$7)$} & 68.9 & 101.2 & 132.6 & 202.8 \\
\hline$(10)$ & 76.8 & 106.3 & 134.4 & 195.8 \\
\hline
\end{tabular}

\subsection{Comparison of Two Binomials}

Consider two binomial processes with $P$ and $P^{*}$ as the values of true proportions and let $p$ and $p^{*}$ be the observed proportions in a sample of $N$ from each process. We have here $H_{1}: P^{*}-P=0$ and $H_{2}: P^{*}-P>0$. The test function $X=p^{*}-p$ under $H_{1}$ has an unspecified variance $V(X)=2[\hat{P}(1-\hat{P})] / N$, where $\hat{P}$ is the common (unspecified) value of $P$ and $P^{*}$ under $H_{1}$. Under $H_{2}$ we have $V(X)=P(1-P) /$ $N+\left[P^{*}\left(1-P^{*}\right)\right] / N$, where $P^{*}-P=\xi>0$ say, with the value of $\xi$ specified but not the values of $P^{*}$ and $P$. We are then faced with the problem of comparing two means having unspecified and unequal variances under $H_{1}$ and $H_{2}$.

The only satisfactory solution to the problem of the comparison of two binomial means-see [4, chapter 7] -is usually given in terms of the transformed variables (section 4.1). Under the inverse sine transformation $\sin ^{-1} \sqrt{p^{*}}-\sin ^{-1} \sqrt{p}$ is approximately normally distributed (except when $N$ is very small or the $P^{\prime}$ 's are close to 0 or 1 ) with mean $\sin ^{-1}$

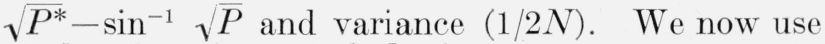
results of section 3 and obtain

$$
\begin{aligned}
N & \cong \frac{1}{2}\left(\frac{K_{\alpha}+K_{\beta}}{\sin ^{-1} \sqrt{P^{*}}-\sin ^{-1} \sqrt{P}}\right)^{2} . \\
A & \cong \frac{K_{\alpha}}{\sqrt{2 N}} \\
K_{\beta} & \simeq \sqrt{2 N}\left(\sin ^{-1} \sqrt{P^{*}}-\sin ^{-1} \sqrt{P}\right)-K_{\alpha} .
\end{aligned}
$$

\section{Tests Relating to the Parameter of the Poisson Distribution}

\subsection{Single Poisson}

Let $\bar{x}$ denote the mean of a random sample of size $N$ from a Poisson population with parameter $m$. Let $H_{1}: m=m_{1}$ and $H_{2}: m=m_{2}\left(m_{2}>m_{1}\right)$. To apply 
the results of Section 3 we assume normality for $\bar{x}$ with mean $m$ and variance $m / N$ and obtain

$$
\begin{aligned}
N & \cong\left(\frac{\sqrt{m_{1}} K_{\alpha}+\sqrt{m_{2}} K_{\beta}}{m_{2}-m_{1}}\right)^{2} \\
A & \cong \frac{m_{2} \sqrt{m_{1}} K_{\alpha}+m_{1} \sqrt{m_{2}} K_{\beta}}{\sqrt{m_{1}} K_{\alpha}+\sqrt{m_{2}} K_{\beta}} \\
K_{\beta} & \cong \frac{\left(m_{2}-m_{1}\right) \sqrt{N}-\sqrt{m_{1}} K_{\alpha}}{\sqrt{m_{2}}}
\end{aligned}
$$

We notice that under both $H_{1}$ and $H_{2}$ the variance of $\bar{x}$ is known given $N$ but differs for $H_{1}$ and $H_{2}$. If we make use of the well known square-root transformation [17], the variance of the transformed variate is approximately independent of the unknown mean and is approximately equal to $1 / 4 N$. We now obtain

$$
\begin{aligned}
& N \cong \frac{1}{4}\left(\frac{K_{\alpha}+K_{\beta}}{\sqrt{m_{2}}-\sqrt{m_{1}}}\right)^{2} \\
& A \cong \frac{K_{\alpha} \sqrt{m_{2}}+K_{\beta} \sqrt{m_{1}}}{K_{\alpha}+K_{\beta}}
\end{aligned}
$$

and

$$
K_{\beta} \cong 2 \sqrt{N}\left(\sqrt{m_{2}}-\sqrt{m_{1}}\right)-K_{\alpha} .
$$

For example for $m_{1}=3.0, m_{2}=4.0, \alpha=.05, \beta=.10$, formulas (16) and (19) yield $N=29.3$ and $N=29.8$, respectively. This is just an illustration; otherwise remarks made in connection with the single binomial in the last paragraph of section 4.1 apply here as well.

\subsection{Comparison of Two Poissons}

Let $M$ and $M^{*}$ be the parameters of two Poisson populations. Let $H_{1}: M=\mathrm{M}^{*}$ and $H_{2}: M<M^{*}$, e.g. $\sqrt{M^{*}}-\sqrt{M}=\eta>0$. Consider two independent random samples of size $N$ drawn one from each of the two populations. Let $\bar{x}$ and $\bar{x}^{*}$ be the corresponding sample means. We may regard the quantity $\sqrt{\bar{x}^{*}}-\sqrt{\bar{x}}$ as approximately normally distributed with mean $m=\sqrt{M^{*}}-\sqrt{M}$ and variance $1 / 2 N$ and consequently obtain

$$
\begin{aligned}
& N=\frac{1}{2}\left(\frac{K_{\alpha}+K_{\beta}}{\sqrt{M^{*}}-\sqrt{M}}\right)^{2} \\
& A \cong \frac{K_{\alpha}}{\sqrt{2 N}}
\end{aligned}
$$

and

$$
K_{\beta} \cong \sqrt{2 N}\left(\sqrt{M^{*}}-\sqrt{M}\right)-K_{\alpha} .
$$

\section{Tests Concerning the Mean of the Normal Population}

\subsection{Single Mean Test ( $\sigma$ known)}

Let $\bar{x}$ denote the mean of a random sample of $N$ observations from a normal population $\left(\mu, \sigma^{2}\right)$. We shall assume that $\sigma$ is known from past experience.
Our hypotheses are $H_{1}: \mu=\mu_{1}$ and $H_{2}: \mu=\mu_{2}\left(\mu_{2}>\mu_{1}\right)$. Set $\mu_{2}-\mu_{1}=\Delta \sigma$. The test function $\bar{x}$ is normally distributed with mean $\mu$ and $\operatorname{sd} \sigma / \sqrt{N}$. Using results of Section 3 we obtain

$$
\begin{gathered}
N=\left(\frac{K_{\alpha}+K_{\beta}}{\Delta}\right)^{2} \\
A=\frac{K_{\alpha} \mu_{2}+K_{\beta} \mu_{1}}{K_{\alpha}+K_{\beta}}
\end{gathered}
$$

and

$$
K_{\beta}=\Delta \sqrt{N}-K_{\alpha} .
$$

\subsection{Comparison of Two Normal Means ( $\sigma^{\prime}$ s known)}

Let $\bar{x}_{1}, \bar{x}_{2}$ be the sample means of two independent random samples of equal size $N$ drawn one from each of the two normal populations $\pi_{1}\left(\mu_{1}, \sigma_{1}^{2}\right), \pi_{2}\left(\mu_{2}, \sigma_{2}^{2}\right)$ respectively. We assume both $\sigma_{1}^{2}$ and $\sigma_{2}^{2}$ to be known from past experience. Our hypotheses are $H_{1}: \mu_{1}=\mu_{2}$ and $H_{2}: \mu_{1}<\mu_{2}$. The test function $\bar{x}_{2}-\bar{x}_{1}$ is normally distributed $\left[\left(\mu_{2}-\mu_{1}\right),\left(\sigma_{1}^{2}+\sigma_{2}^{2}\right) / N\right]$ and, therefore, we obtain

$$
\begin{gathered}
N=\left(\frac{K_{\alpha}+K_{\beta}}{d}\right)^{2} \\
A=\frac{K_{\alpha}\left(\mu_{2}-\mu_{1}\right)}{K_{\alpha}+K_{\beta}}
\end{gathered}
$$

and

$$
K_{\beta}=\sqrt{N} d-K_{\alpha}
$$

where

$$
d=\frac{\mu_{2}-\mu_{1}}{\sqrt{\sigma_{1}^{2}+\sigma_{2}^{2}}} .
$$

If $\sigma_{1}^{2}=\sigma_{2}^{2}=\sigma^{2}$ we obtain

$$
\begin{gathered}
N=2\left(\frac{K_{\alpha}+K_{\beta}}{\Delta}\right)^{2} \\
A=\frac{K_{\alpha}\left(\mu_{2}-\mu_{1}\right)}{K_{\alpha}+K_{\beta}}
\end{gathered}
$$

and

$$
K_{\beta}=\Delta \sqrt{\frac{N}{2}}-K_{\alpha} .
$$

\subsection{Single Mean Test ( $\sigma$ unknown)}

For notation see section 6.1 . In this case the well known Student's statistic $t=\sqrt{N}\left(\bar{x}-\mu_{1}\right) / s$ where $s^{2}$ is the unbiased estimate of $\sigma^{2}$, is used to accept or reject $H_{1}$. Tables for determining the sample size for detecting a given value of $\Delta$ with preassigned $\alpha$ and $\beta$ are given in [1] and operating characteristic curves for the same are given in [2]. To obtain a convenient formula for the sample size we proceed as follows:

Consider $P\{t \geq k\}=P\{[(\bar{x}-\mu)-(k s / \sqrt{N})]>0\}$ where $k$ is a certain constant. The quantity $Z=$ $\left(\bar{x}-\mu_{1}\right)-(k s / \sqrt{N})$ consists of two parts: $\left(\bar{x}-\mu_{1}\right)$ is normally distributed $\left(0, \sigma^{2} / N\right)$ under $H_{1}$, and 
$\left(\mu_{2}-\mu_{1}, \sigma^{2} / N\right)$ under $H_{2}$; and for fixed $N, k s / \sqrt{N}$ is a constant multiple of $s$ where $s$ is approximately normally distributed $\left(C_{1} \sigma, C_{2} \sigma / \sqrt{2(N-1)}\right)$ and $C_{1}$ and $C_{2}$ are certain constants less than $1 .{ }^{4}$ It will be assumed for the purpose of this discussion that both $C_{1}$ and $C_{2}$ are equal to unity. The dual nature of these assumed approximations to the distribution of $s$ should be noted. Therefore,

$$
\begin{aligned}
E\left(Z \mid H_{1}\right) & =Z_{1}=-\frac{k}{\sqrt{N}} \sigma \\
E\left(Z \mid H_{2}\right) & =Z_{2}=\left(\mu_{2}-\mu_{1}\right)-\frac{k}{\sqrt{N}} \sigma \\
V(Z) & =\frac{\sigma^{2}}{N}+\frac{k^{2}}{N} \cdot \frac{\sigma^{2}}{2(N-1)} .
\end{aligned}
$$

For $Z>A$ as the critical region we obtain (see section 3 )

$$
\begin{aligned}
& \frac{A-\left(-k \frac{\sigma}{\sqrt{N}}\right)}{\frac{\sigma}{\sqrt{N}} \sqrt{1+\frac{k^{2}}{2(N-1)}}}=K_{\alpha} \\
& \frac{A-\left(\mu_{2}-\mu_{1}-\frac{k}{\sqrt{N} \sigma)}\right.}{\frac{\sigma}{\sqrt{N}} \sqrt{1+\frac{k^{2}}{2(N-1)}}}=-K_{\beta}
\end{aligned}
$$

Eliminating $A$ from the above two equations we get

$$
\frac{\Delta}{K_{\alpha}+K_{\beta}}=\frac{1}{\sqrt{N}} \sqrt{1+\frac{k^{2}}{2(N-1)}}
$$

where $\Delta$ has been previously defined (section 6.1 ). This is a quadratic in $N$ and could be solved for $N$ if $k$ were known. We now determine $k$ from the consideration that $A$ is to be independent of $\sigma$. After simplification we obtain

$$
A=\sigma\left(\frac{\Delta K_{\alpha}}{K_{\alpha}+K_{\beta}}-\frac{k}{\sqrt{N}}\right)
$$

The right-hand side will be independent of $\sigma$ if and only if the quantity in parentheses vanishes.

${ }_{4}$ The general expressions for $C_{1}$ and $C_{2}$ are:

$$
C_{1}=\frac{\sqrt{\frac{2}{N-1}} \Gamma\left(\frac{N}{2}\right)}{\Gamma\left(\frac{N-1}{2}\right)}, \quad C_{2}=\sqrt{2(N-1)\left(1-C_{1}^{2}\right)} .
$$

For small values of $N, C_{1}$, and $C_{2}$ take the following values:

\begin{tabular}{|r|r|r|}
\hline \multirow{2}{*}{$N$} & \multicolumn{1}{|c|}{$C_{1}$} & \multicolumn{1}{c|}{$C_{2}$} \\
\cline { 1 - 1 } & \multicolumn{1}{r|}{} & \\
\cline { 1 - 2 } 2 & 0.798 & 0.852 \\
3 & .886 & .927 \\
4 & .921 & .954 \\
5 & .940 & .965 \\
10 & .973 & .985 \\
25 & .990 & .995 \\
& & \\
\hline
\end{tabular}

Therefore,

$$
k=\frac{K_{\alpha} \Delta \sqrt{N}}{K_{\alpha}+K_{\beta}}
$$

whence $A=0$ and is not only independent of $\sigma$ but actually takes the originally intended value in the inequality $P(Z>0)$. Substituting this value of $k$ in (34) we finally obtain

$$
N=\frac{b+\sqrt{b^{2}-4 a}}{2 a}
$$

where

$$
a=\left(\frac{\Delta}{K_{\alpha}+K_{\beta}}\right)^{2}
$$

and

$$
b=\left(1+a\left(1+\frac{K_{\alpha}^{2}}{2}\right)\right)
$$

Similarly

$$
K_{\beta}=-K_{\alpha}+\frac{\Delta}{\sqrt{N-1}}\left(N^{2}-N\left(1+\frac{K_{\alpha}^{2}}{2}\right)\right)^{1 / 2}
$$

If we replace $N-1$ by $N$, the above two formulas reduce to somewhat simpler expressions of the form

$$
\begin{gathered}
N=\left(\frac{1}{a}+\frac{1}{2} K_{\alpha}^{2}\right)+1 \\
K_{\beta}=-K_{\alpha}+\Delta\left(N-\frac{K_{\alpha}^{2}}{2}\right)^{1 / 2} .
\end{gathered}
$$

For any given $\Delta, \alpha$, and $\beta$, values of $N$ from (36) are compared with the Neyman-Tokarska ${ }^{5}$ Tables [1] are given in table 1 . These values will be found to be approximately the same.

As pointed out in the previous paragraph (34) was obtained under the assumption that $k$ is unknown. The classical procedure employs $k=t_{\alpha}(n)$ where $n=N-1 .^{5} \quad$ Therefore if the probability points of $t$ are not available, (35) furnishes an approximation to such points $t_{\alpha}$. We have considered the accuracy of such $k$ points in relation to $t_{\alpha}$ in terms of $P[t>x]=\alpha(x)$. Values of $t_{\alpha}, k, \alpha\left(t_{\alpha}\right)$, and $\alpha(k)$ are given in tables $2 \mathrm{a}$ and $2 \mathrm{~b}$ for different $N$ 's and for different $\alpha$ and $\beta$. Strictly speaking $\alpha\left(t_{\alpha}\right) \equiv \alpha$, but when $t_{\alpha}$ to only three decimals is used, $\alpha\left(t_{\alpha}\right)$ may differ slightly from $\alpha$ as shown in tables $2 \mathrm{a}$ and $2 \mathrm{~b}$.

We notice that $k$ values are in general conservative $t_{\alpha}$ estimators and that the values of $a(k)$ are consistently greater than the corresponding value of $a\left(t_{\alpha}\right)$. In this sense a user of our formulas is likely to declare slightly too many significant results. The danger, if it can be so called, is not very great, but it is still there.

The question is asked: is it possible to eliminate this "danger" and still utilize formula (36) for $N$ ? It should be noticed that we can not utilize the available percentage points of $t$ in the derivation of (36). However, (34) can still be solved for $N$ as-

5 Neyman-Tokarska's $\rho$ is equal to our $\Delta \sqrt{N}$. Since the standard table of ${ }^{5}$ Neyman-Tokarska's $\rho$ is equal to our $\Delta \sqrt{N}$. Since the standard table of
probability points of $t,[18]$ table 4 , gives the two-tail probability points of $t$, our probability points of $t$, [18] table 4 , gives the two-tail probability points of $t$,
$t_{\alpha}(n)$ corresponds to the entry given there for $2 \alpha$ and $n$ degrees of freedom. 
TABLE 1. Comparisons among different methods for obtaining necessary sample sizes

[Single sample one-sided mean test]

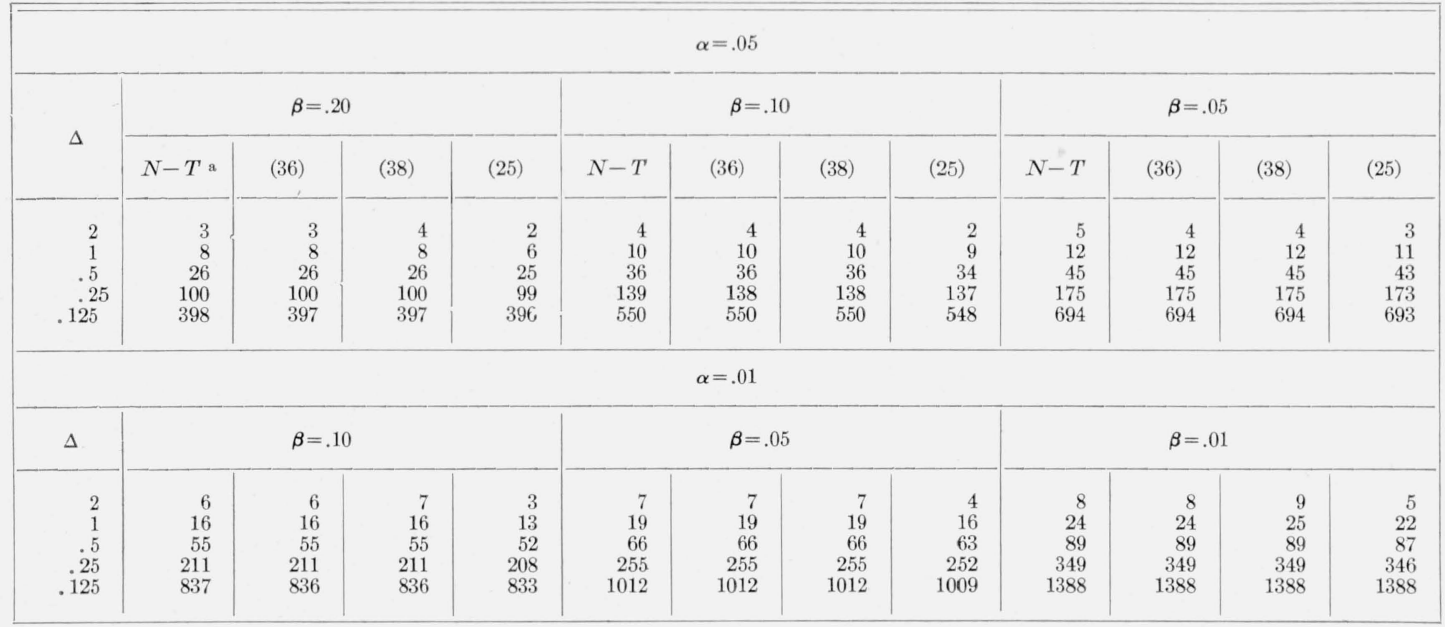

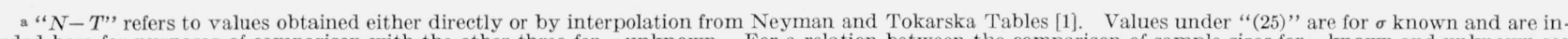

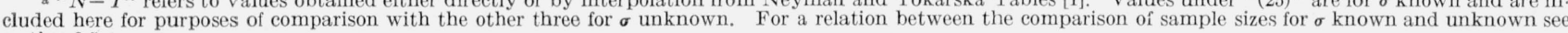
section 6.5 .

TaBle 2a. ${ }^{\text {a }}$ Comparison of the accuracy of the different percentage points of $t$ and $k$ values for the same $\Delta$

\begin{tabular}{|c|c|c|c|c|c|c|}
\hline & \multicolumn{6}{|c|}{$\alpha=.05$} \\
\hline & \multicolumn{3}{|c|}{ Neyman and Tokarska } & \multicolumn{3}{|c|}{ This paper } \\
\hline & $N$ & $t_{\alpha}$ & $\alpha\left(t_{\alpha}\right)$ & $N$ & $k$ & $\alpha(k)$ \\
\hline$\beta=.50$ & $\left\{\begin{array}{r}3 \\
4 \\
12\end{array}\right.$ & $\begin{array}{l}\text { 2. } 920 \\
2.353 \\
\text { 1. } 796\end{array}$ & $\begin{array}{r}0.05000 \\
.05000 \\
.05003\end{array}$ & $\begin{array}{r}3 \\
4 \\
12\end{array}$ & $\begin{array}{l}\text { 3. } 338 \\
2.110 \\
1.753\end{array}$ & $\begin{array}{r}0.04958 \\
.06270 \\
.05370\end{array}$ \\
\hline$\beta=.20$ & $\left\{\begin{array}{r}3 \\
8 \\
26\end{array}\right.$ & $\begin{array}{l}2.920 \\
1.895 \\
1.708\end{array}$ & $\begin{array}{l}.05000 \\
.04996 \\
.05012\end{array}$ & $\begin{array}{r}3 \\
8 \\
26\end{array}$ & $\begin{array}{l}\text { 2. } 458 \\
\text { 1. } 840 \\
\text { 1. } 691\end{array}$ & $\begin{array}{r}.06659 \\
.05412 \\
.05163\end{array}$ \\
\hline$\beta=.10$ & $\left\{\begin{array}{r}4 \\
10\end{array}\right.$ & $\begin{array}{l}\text { 2. } 353 \\
\text { 1. } 833\end{array}$ & $\begin{array}{l}.05000 \\
.04999\end{array}$ & $\begin{array}{r}4 \\
10\end{array}$ & $\begin{array}{l}\text { 2. } 235 \\
\text { 1. } 783\end{array}$ & $\begin{array}{l}.05575 \\
.05414\end{array}$ \\
\hline$\beta=.05$ & $\left\{\begin{array}{r}5 \\
12\end{array}\right.$ & $\begin{array}{l}\text { 2. } 132 \\
\text { 1. } 796\end{array}$ & $\begin{array}{l}.04995 \\
.05003\end{array}$ & $\begin{array}{r}4 \\
12\end{array}$ & $\begin{array}{l}2.110 \\
1.753\end{array}$ & $\begin{array}{l}.06270 \\
.05370\end{array}$ \\
\hline$\beta=.01$ & $\left\{\begin{array}{r}6 \\
17\end{array}\right.$ & $\begin{array}{l}\text { 2. } 015 \\
\text { 1. } 746\end{array}$ & $\begin{array}{l}.05003 \\
.04995\end{array}$ & $\begin{array}{r}6 \\
17\end{array}$ & $\begin{array}{l}\text { 1. } 959 \\
\text { 1. } 718\end{array}$ & $\begin{array}{l}.05370 \\
.05250\end{array}$ \\
\hline
\end{tabular}

2 Values of $P(t>k)=\alpha(k)$ and $P\left(t>t_{\alpha}\right)=\alpha\left(t_{\alpha}\right)$ for $N \leq 21$ were obtained by interpolation in "Student's" Table $I$ [ 6$]$ and for $22 \leq N \leq 31$ by interpolation in Table XXV of $[7]$.

suming $k$ to be known, say equal to $t_{\alpha}(n)$, resulting in

$$
N=\frac{(a+1)+\sqrt{(a-1)^{2}+2 a t_{\alpha}^{2}(n)}}{2 a}
$$

Values of $N$ from (38) corresponding to different $t_{\alpha}(n), \Delta, a$ and $\beta$ are also given in table 1 . Since the values of $N$ as given by (36), (38) and the NeymanTokarska Tables are approximately the same, it is recommended that (38) be used in conjunction with the $t$-tables. This will save the labor of calculating $k$ values.

Since any symmetric two-sided test may be regarded as a combination of two one-sided tests, values of $N$ and $k$ may be obtained from the corresponding single-tailed formulas by substituting $K_{\alpha} / 2$ for $K_{\alpha}$ and $t_{\alpha} / 2$ for $t_{\alpha}$, in which case the " $\beta$ " deduced from the resulting value of $K_{\beta}$ will over-estimate
TABLE 2b. ${ }^{a}$ Comparison of the accuracy of the different percentage points of $t$ and $k$ values for the same $\Delta$

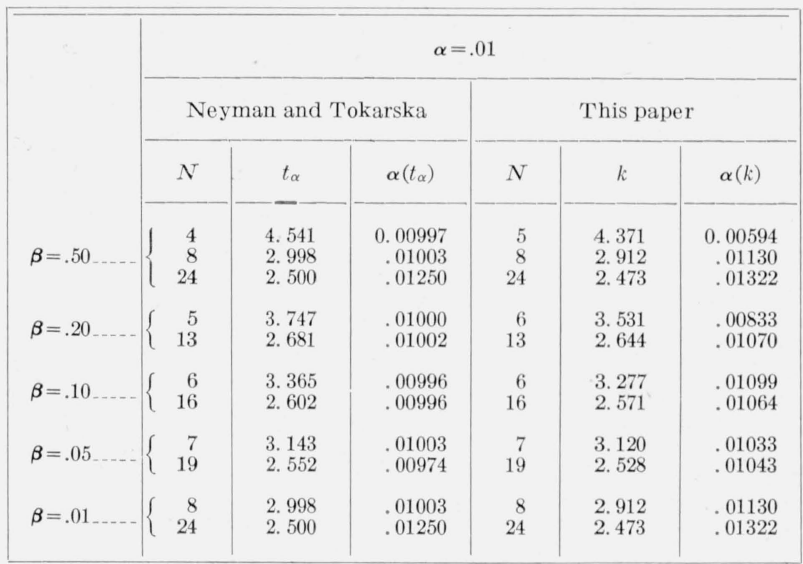

a Values of $P(t>k)=\alpha(k)$ and $P\left(t>t_{\alpha}\right)=\alpha\left(t_{\alpha}\right)$ for $N \leq 21$ were obtained by interpolation in "Student's" Table I [6] and for $22 \leq N \leq 31$ by interpolation in Table XXV of [7].

the true value of $\beta$, the probability of accepting $H_{1}$ when $\mu_{2}-\mu_{1}=\Delta \sigma$, by the amount $1-\beta^{\prime}$ where $\beta^{\prime}$ is given by (37) or (37a) with the term in $\Delta$ taken with a negative sign. Values of $N$ obtained from [8] and [9] and from the formulas (36) and (38) of this paper are given in table 3 . The corresponding twotailed values of $t$ and $k$ are also given in table 3 .

\subsection{Comparison of Two Means: (common $\sigma$ un- known)}

For notation refer to section 6.2. Consider two samples of equal size $N$. Our hypotheses are $H_{1}$ : $\mu_{1}=\mu_{2} ; H_{2}: \mu_{1}<\mu_{2}$. Let $s^{2}$ denote the unbiased estimate of the common variance $\sigma^{2}$. The statistic $t=\frac{\bar{x}_{2}-\bar{x}_{1}}{\sqrt{\frac{2}{N}}}$ which under $H_{1}$ has "Student's" $t$-distribu- 
TABLE 3. Comparisons among different methods for obtaining necessary sample sizes

[Two-sided single mean test: $\sigma$ unknown]

\begin{tabular}{|c|c|c|c|c|c|c|c|c|c|c|}
\hline \multicolumn{11}{|c|}{$\alpha=.05$} \\
\hline \multirow{2}{*}{$\Delta$} & \multicolumn{5}{|c|}{$\boldsymbol{\beta}=.30$} & \multicolumn{5}{|c|}{$\beta=.20$} \\
\hline & $\begin{array}{l}\text { Tabu- } \\
\text { lated }\end{array}$ & $(36)$ & (38) & $t_{\alpha} / 2$ & $k$ & $\begin{array}{l}\text { Tabu- } \\
\text { lated }\end{array}$ & $(36)$ & (38) & $t_{\alpha} / 2$ & $k$ \\
\hline $\begin{array}{r}2 \\
1 \\
.5 \\
.25 \\
.125\end{array}$ & $\begin{array}{r}4 \\
8 \\
27 \\
101 \\
397\end{array}$ & $\begin{array}{r}4 \\
8 \\
27 \\
101 \\
397\end{array}$ & $\begin{array}{r}4 \\
8 \\
27 \\
101 \\
397\end{array}$ & $\begin{array}{l}3.182 \\
2.365 \\
2.056 \\
1.982 \\
1.966\end{array}$ & $\begin{array}{l}3.190 \\
2.280 \\
2.038 \\
1.979 \\
1.965\end{array}$ & $\begin{array}{r}4 \\
10 \\
33 \\
128 \\
504\end{array}$ & $\begin{array}{r}4 \\
10 \\
33 \\
128 \\
504\end{array}$ & $\begin{array}{r}5 \\
10 \\
33 \\
128 \\
504\end{array}$ & $\begin{array}{l}3.182 \\
2.262 \\
2.037 \\
1.977 \\
1.964\end{array}$ & $\begin{array}{l}2.949 \\
2.210 \\
2.021 \\
1.975 \\
1.964\end{array}$ \\
\hline \multicolumn{11}{|c|}{$\alpha=.01$} \\
\hline $\begin{array}{r}2 \\
1 \\
.5 \\
.25 \\
.125\end{array}$ & $\begin{array}{r}6 \\
13 \\
42 \\
157 \\
618\end{array}$ & $\begin{array}{r}6 \\
13 \\
42 \\
157 \\
618\end{array}$ & $\begin{array}{r}6 \\
13 \\
42 \\
157 \\
618\end{array}$ & $\begin{array}{l}4.032 \\
3.055 \\
2.701 \\
2.607 \\
2.584\end{array}$ & $\begin{array}{l}4.184 \\
3.019 \\
2.687 \\
2.604 \\
2.583\end{array}$ & $\begin{array}{r}6 \\
15 \\
50 \\
190 \\
751\end{array}$ & $\begin{array}{r}7 \\
15 \\
50 \\
190 \\
751\end{array}$ & $\begin{array}{r}7 \\
15 \\
50 \\
190 \\
751\end{array}$ & $\begin{array}{l}\text { 4. } 032 \\
\text { 2. } 977 \\
\text { 2. } 680 \\
\text { 2. } 601 \\
\text { 2. } 583\end{array}$ & $\begin{array}{l}3.933 \\
2.941 \\
2.668 \\
2.599 \\
2.582\end{array}$ \\
\hline
\end{tabular}

a "Tabulated" values were obtained from [8] and [9] and $t_{\alpha} / 2$ indicates the two-tailed $\alpha$ value of $t$. See footnote 5 .

tion with $2(N-1)$ d.o.f. is used to accept or reject $H_{1}$. Consider $P\left(t \geq \mathrm{k}_{1}\right)=\mathrm{P}\left(\bar{x}_{2}-\bar{x}_{1}-k_{1} \sqrt{\frac{2}{N}} s>0\right)$ where we assume $k_{1}$ to be a certain unknown constant. Let

$$
Z=\left(\bar{x}_{2}-\bar{x}_{1}\right)-k_{1} \sqrt{\frac{2}{N}} s .
$$

We assume that $s$ is approximately normally distributed with mean $\sigma$ and sd, $\sigma / \sqrt{4(N-1)}$. Therefore $Z$ is approximately normally distributed with

$$
\begin{gathered}
E\left(Z \mid H_{1}\right)=-k_{1} \sqrt{\frac{2}{N}} \sigma \\
E\left(Z \mid H_{2}\right)=\mu_{2}-\mu_{1}-k_{1} \sqrt{\frac{2}{N}} \sigma \\
\sigma(Z)=\sigma \sqrt{\frac{2}{N}+\frac{k_{1}^{2}}{2 N(N-1)}}
\end{gathered}
$$

where $\sigma(Z)$ denotes the standard deviation of $Z$. Proceeding as in section 3.3 we obtain

and

$$
\frac{\Delta}{K_{\alpha}+K_{\beta}}=\sqrt{\frac{2}{N}+\frac{k_{1}^{2}}{2 N(N-1)}}
$$

$$
A=\frac{K_{\alpha} \sigma \Delta}{K_{\alpha}+K_{\beta}}-\sigma \sqrt{\frac{2}{N}} k_{1} .
$$

If we assume $k_{1}=t_{\alpha}(n)$ where $t_{\alpha}$ is the one-sided $\alpha$-point of $t$ for $n=2(N-1)$ d.o.f the equation (39) yields

$$
N=\frac{a+2+\sqrt{(a-2)^{2}+2 a t_{\alpha}^{2}(n)}}{2 a}
$$

where $a$ has been defined in section 6.3 . The reason for such an illogical assumption about the knowledge of $t_{\alpha}(n)$ before actually $N$ is determined has been indicated in the previous section. If $k_{1}$ is un- known we determine $k_{1}$ from the consideration that $A$ is to be independent of $\sigma$. The relation (40) yields

$$
k_{1}=\sqrt{\frac{\bar{N}}{2}} \cdot \frac{K_{\alpha} \Delta}{K_{\alpha}+K_{\beta}} .
$$

We substitute this value of $k_{1}$ in (39) and obtain

$$
N=\frac{b_{1}+\sqrt{b_{1}^{2}-8 a}}{2 a}
$$

where $a$ has been previously defined (section 6.3) and

$$
b_{1}=2+a\left(1+\frac{K_{\alpha}^{2}}{4}\right) \text {. }
$$

For determining the operating characteristic we

\begin{tabular}{|c|c|c|c|c|}
\hline \multirow{7}{*}{$\begin{array}{l}\beta=.50 \\
\beta=.20 \\
\beta=.10 \\
\beta=.05 \\
\beta=.01\end{array}$} & \multicolumn{2}{|c|}{$\alpha=.05$} & \multicolumn{2}{|c|}{$\alpha=.01$} \\
\hline & \multicolumn{2}{|c|}{$\underset{(43)}{\text { Neyman-Tokarska }_{(43)}}$} & \multicolumn{2}{|c|}{$\underset{(43)}{\text { Neyman-Tokarska }}$} \\
\hline & 2 & 2 & 4 & 4 \\
\hline & 4 & 4 & 6 & $\begin{array}{l}4 \\
7\end{array}$ \\
\hline & 5 & 5 & 8 & 8 \\
\hline & 6 & 6 & 9 & 9 \\
\hline & 8 & 9 & 12 & $\begin{array}{r}5 \\
12\end{array}$ \\
\hline
\end{tabular}
similarly obtain

$$
K_{\beta}=-K_{\alpha}+\Delta\left[\frac{N}{2}\left(1-\frac{K_{\alpha}^{2}}{4(N-1)}\right)\right]^{1 / 2} .
$$

We give in table 4 values of $N$ as obtained from (43) and Tables of Neyman ${ }^{6}$ and Tokarska [1]. While (42) provides approximate values of the per-

TABLE 4. Comparisons among different methods for obtaining necessary sample sizes $N^{\text {s }}$

[Two means one-sided test; common $\sigma$ unknown: $\Delta=2$ ]

a $N$ is the size of one of the two equal samples.

${ }^{6}$ For this particular case $\rho$ as defined in [1] is equal to $\Delta \sqrt{\frac{N}{2}}$. 
centage points of $t$ for $2(N-1)$ d.o.f, the formula (43) may be used advantageously by utilizing the available percentage points of $t$ as acceptance-rejection criteria. Formulas obtained here for single-tailed comparisons can also be used for a two-tailed test by substituting appropriate two-tailed values of the quantities involved. If the two populations have unequal variances and their ratio is known we still can construct a test function similar to $t$ and use the above formulas. (For the structure of the test function see [10].)

\subsection{Comparison of Sample Sizes for Known and Unknown $\sigma$}

It need hardly be emphasized that in situations in which large sample sizes are required, the normal test (sections 6.1 and 6.2) and $t$-test (sections 6.3 and 6.4) will both yield approximately the same $N$ (see for example table 1). To determine the relations between the two, let $N_{m}$ be the number of observations required when $\sigma$ is known and $N_{t}$ the corresponding number when $\sigma$ is not known. After some simplification the following asymptotic relations between $N_{m}$ and $N_{t}$ are obtained. For the single-mean test, (25) and (36a) yield

$$
N_{t} \sim N_{m}\left(1+\frac{K_{\alpha}^{2}}{2 N_{m}}\right)+1
$$

and for the two-means test, (31) and (43) yield

$$
N_{t} \sim N_{m}\left(1+\frac{K_{\alpha}^{2}}{4 N_{m}}\right)+1
$$

\section{Tests Concerning Variances of Normal Populations}

Tests relating to population variances fall into two well defined categories. In one case we assume that the variability of a certain product is known and it is desired to find out whether a new product is more variable than this. In the other case we are asked to choose between two products on the basis of their variability which is unknown. We discuss these situations in the following sections.

\subsection{Single Variance Test}

Consider a random sample of size $N(=n+1)$ from a normal population $\left(m, \sigma^{2}\right)$. Let $s^{2}$ be the unbiased sample estimate of $\sigma^{2}$. Our hypotheses are $H_{1}$ : $\sigma^{2}=\sigma_{0}^{2}$ and $H_{2}: \sigma^{2}=\lambda \sigma_{0}^{2}(\lambda>1)$. For a given level of significance $\alpha$, if $\chi^{2}=n s^{2} / \sigma_{0}^{2}>\chi_{\alpha}^{2}$, we reject $H_{1}$ and conclude that $\sigma^{2}>\sigma_{0}^{2}$.

Let $\lambda(\alpha, \beta, n)^{7}$ denote the value of $\sigma^{2} / \sigma_{0}^{2}$ for which the probability of the decision $\sigma^{2}=\sigma_{0}^{2}$ equals $\beta$ when the test is conducted at the $\alpha$ level of significance with $n$ d.o.f. It can be shown [2], [4] that the probability error of the second kind is exactly $\beta$ if $\lambda(\alpha, \beta, n)=$ $\left.\chi_{\alpha}^{2}(n) / \chi_{1-\beta}^{2}(n)\right)$. If we are testing $H_{1}: \sigma^{2}=\sigma_{0}^{2}$ against $H_{2}: \quad \sigma^{2}=\lambda \sigma_{0}^{2} \quad(\lambda<1) \quad$ we have $\lambda(\alpha, \beta, n)=x_{1-\alpha}^{2} / \chi_{\beta}^{2}$.

\footnotetext{
7 Our $\lambda(\alpha, \beta, n)$ is equivalent to $\rho(\alpha, \beta, n)$ of [4].
}

Curves for the operating characteristics of such testing procedures are given in [2] and [4]. Eisenhart [4] has also given extensive tables for $\lambda(\alpha, \beta, n)$.

The problem of determining a direct relation between large $n$ and any given set of values of $\lambda, \alpha, \beta$ was first considered by Wallis $[1$, footnote of $p$. 278]. Assuming normality of $s$ (see section 6.3) and applying the results of Section 3 we obtain

$$
\begin{gathered}
n=\frac{1}{2}\left(\frac{K_{\alpha}+K_{\beta} \sqrt{\lambda}}{\sqrt{\lambda}-1}\right)^{2} \\
A=\frac{\sqrt{\lambda} \sigma_{0}\left(K_{\alpha}+K_{\beta}\right)}{K_{\alpha}+\sqrt{\lambda} K_{\beta}} \\
\lambda(\alpha, \beta, n)=\left(\frac{K_{\alpha}+\sqrt{2 n}}{\sqrt{2 n}-K_{\beta}}\right)^{2}
\end{gathered}
$$

To compare the accuracy of this formula with the Tables [4] consider the following situation: if a decision $\sigma=\sigma_{0}$ is a serious error from the practical viewpoint when $\sigma=1.500 \sigma_{0}$ and it is desired to keep the risk of such an error below .05 when the test is conducted at the 5-percent level of significance, how many d.o.f. will be needed for $s^{2}$ ? The formula (47) gives $n=33.8$ and from [4] we find that 34 d.o.f. are needed. Table 5 presents the calculated values of $n$ from (47) and table 6 presents the calculated values of $\lambda$ from (49). For a comparative discussion on the use of these formulas in relation to others see next section.

\subsection{An Alternative Formula for the Single Variance Test Based on the Distribution of Log $s^{8}$}

As pointed out by Bartlett and Kendall [11] the distribution of $\log s^{2}$ depends on $\sigma^{2}$ only through the term $\sigma^{2}$ in its expected value. Consequently the choice of the critical region based on the distribution of $\log s$ in place of $s$ has obvious advantages. In this section we explore the possibility of using some formulas based on the distribution of $\log s$.

The cumulant function $\mathrm{K}(t)$ of $\log s$ is given [11] by

$\mathrm{K}(t)=\frac{i t}{2}\left(\log \sigma^{2}-\log \frac{n}{2}\right)+\log \Gamma\left(\frac{n+i t}{2}\right)-\log \Gamma\left(\frac{n}{2}\right)$

which yields the following expressions for the first two cumulants

$$
\begin{aligned}
& \kappa_{1}=\frac{1}{2}\left(\log \sigma^{2}-\log \frac{n}{2}\right)+\frac{1}{2} \Psi\left(\frac{n}{2}\right) \\
& \kappa_{2}=\frac{1}{4} \Psi^{\prime}\left(\frac{n}{2}\right),
\end{aligned}
$$

where $\Psi(x)=\frac{d}{d x} \log \Gamma(x)$. The results of Section 3

${ }^{8}$ All logarithms are te the base $e .$. 
applied to $\log s$ yields

$$
\begin{aligned}
\kappa_{2}(n) & =\left(\frac{\log \frac{\sigma}{\sigma_{0}}}{K_{\alpha}+K_{\beta}}\right)^{2} \\
A & =\frac{K_{\beta} \log \sigma_{0}+K_{\alpha} \log \sqrt{\lambda} \sigma_{0}}{K_{\alpha}+K_{\beta}}+\frac{1}{2}\left(\Psi\left(\frac{n}{2}\right)-\log \frac{n}{2}\right)
\end{aligned}
$$

$\lambda(\alpha, \beta, n)=e^{2 \sqrt{\kappa_{2}(n)}\left(K_{\alpha}+K_{\beta}\right)}$.

These formulas assume no other approximation except that of normality and in that sense may be regarded as exact relative to (47), which assumes dual type of approximation for the distribution of $s$ (see section 6.3). While the accuracy of (50) and (52) does not depend upon the accuracy with which we estimate $\kappa_{1}$, it does depend upon the complicated expression $\Psi^{\prime}\left(\frac{n}{2}\right)$. For a given $\alpha, \beta$, and $\lambda$ the only way in which we can utilize (50) for finding the necessary sample size is to approximate the

TABLE 5a. Comparison of the sample sizes $(n=N-1)$ for the

\begin{tabular}{|c|c|c|c|c|c|c|}
\hline \multicolumn{7}{|c|}{$\alpha=.05$} \\
\hline \multirow{2}{*}{$\lambda$} & \multicolumn{3}{|c|}{$\beta=.05$} & \multicolumn{3}{|c|}{$\beta=.10$} \\
\hline & (47) & (53) & $\begin{array}{l}\text { Tabu- } \\
\text { lated }\end{array}$ & (47) & (53) & $\begin{array}{l}\text { Tabu- } \\
\text { lated }\end{array}$ \\
\hline $\begin{array}{l}2 \\
2.5 \\
3 \\
3.5\end{array}$ & $\begin{array}{l}46.0 \\
26.7 \\
18.8 \\
14.7\end{array}$ & $\begin{array}{l}46.1 \\
26.8 \\
18.9 \\
14.8\end{array}$ & $\begin{array}{l}46.4 \\
26.6 \\
18.8 \\
14.6\end{array}$ & $\begin{array}{l}34.9 \\
20.0 \\
13.9 \\
10.8\end{array}$ & $\begin{array}{l}36.7 \\
21.4 \\
15.2 \\
11.9\end{array}$ & $\begin{array}{l}36.0 \\
20.5 \\
14.5 \\
11.1\end{array}$ \\
\hline
\end{tabular}
single variance test

TABle 5b. Comparison of the sample sizes $(n=N-1)$ for the

\begin{tabular}{|c|c|c|c|c|c|c|}
\hline \multicolumn{7}{|c|}{$\alpha=.01$} \\
\hline \multirow{2}{*}{$\lambda$} & \multicolumn{3}{|c|}{$\beta=.01$} & \multicolumn{3}{|c|}{$\beta=.05$} \\
\hline & (47) & (53) & $\begin{array}{l}\text { Tabu- } \\
\text { lated }\end{array}$ & (47) & (53) & $\begin{array}{l}\text { Tabu- } \\
\text { lated }\end{array}$ \\
\hline $\begin{array}{l}2 \\
2.5 \\
3 \\
3.5\end{array}$ & $\begin{array}{l}91.9 \\
53.4 \\
37.7 \\
29.4\end{array}$ & $\begin{array}{l}91.1 \\
52.6 \\
36.9 \\
28.6\end{array}$ & $\begin{array}{l}91.4 \\
53.1 \\
37.6 \\
28.7\end{array}$ & $\begin{array}{l}63.1 \\
35.9 \\
25.0 \\
19.3\end{array}$ & $\begin{array}{l}66.6 \\
38.6 \\
27.1 \\
21.1\end{array}$ & $\begin{array}{l}64.3 \\
37.2 \\
25.6 \\
19.8\end{array}$ \\
\hline
\end{tabular}
single variance test asymptotic expansion of $\Psi^{\prime}\left(\frac{n}{2}\right)[12]$.

As a first approximation if we set $\kappa_{2} \cong 1 /[2(n-1)]$ we obtain

$$
\begin{gathered}
n=1+2\left(\frac{K_{\alpha}+K_{\beta}}{\log \lambda}\right)^{2} \\
\log \lambda=\sqrt{\frac{2}{n-1}}\left(K_{\alpha}+K_{\beta}\right) .
\end{gathered}
$$

In tables 5a and 5b values of $n$ from [4], and values calculated from (47) and (53) for different values of $\alpha, \beta$, and $\lambda$ are compared. In table 6 we give values of $\lambda(\alpha, \beta, n)$ to reemphasize the nature of approximations based on the distribution of $s$ and $\log s$. In this connection for the application of $(52)$ values of $\kappa_{2}(n)$ were taken from [11].

It appears that for the customary values of the probabilities of errors of the two kinds $\alpha=\beta=.05$ and $\alpha=\beta=.01$, formulas (47) and (53) provide very good approximations to $n$ for small sample sizes. If the percentage points of the $\chi^{2}$-distribution are available, (47) is preferable because it is easier to compute $n$ from (47) than from (53). Even for such a small value as $n=5,(47)$ errs on the safe side in this sense that it gives (at least for $\alpha=\beta$ ) a sample size which will be always sufficient to detect this difference. The formula (53) also shares this property with (47). In the absence of the percentage points of the $\chi^{2}$-distribution it perhaps ought to be emphasized that on comparison of the critical regions for $s$ and $\log s$ (cf. (48) and (51)) there is not much basis for choice. The choice of the critical region based on the distribution of $\log s$ has certain theoretical advantages, but the computation of the critical region is somewhat more complicated since it involves the approximation of $\Psi(n / 2)$.

The effectiveness of formulas (47) and (53) varies when $\alpha$ and $\beta$ are not equal. It appears (see table 5 and table 6) that for $\beta>\alpha$ it is safer to use (53) because it is always likely to err on the safe side in the sense of the previous paragraph. However, if $\beta<\alpha$ it appears that it is safer to use (47) because (53) is likely to give a value of $n$, which will actually be less than the necessary sample size.

\subsection{Comparison of Two Population Variances}

\begin{tabular}{|c|c|c|c|c|c|c|c|c|c|c|c|c|}
\hline \multirow{3}{*}{$n$} & \multicolumn{12}{|c|}{$\alpha=.05$} \\
\hline & \multicolumn{4}{|c|}{$\beta=.25$} & \multicolumn{4}{|c|}{$\beta=.05$} & \multicolumn{4}{|c|}{$\beta=.01$} \\
\hline & $(49)$ & $(52)$ & (54) & Tabulated & $(49)$ & $(52)$ & $(54)$ & Tabulated & $(49)$ & $(52)$ & $(54)$ & Tabulated \\
\hline $\begin{array}{r}5 \\
10 \\
15 \\
20\end{array}$ & $\begin{array}{l}3.734 \\
2.595 \\
2.199 \\
1.990\end{array}$ & $\begin{array}{l}5.073 \\
2.977 \\
2.401 \\
2.122\end{array}$ & $\begin{array}{l}5.155 \\
2.984 \\
2.403 \\
2.122\end{array}$ & $\begin{array}{l}4.139 \\
2.717 \\
2.265 \\
2.033\end{array}$ & $\begin{array}{r}10.037 \\
4.681 \\
3.454 \\
2.900\end{array}$ & $\begin{array}{c}10.01 \\
4.700 \\
3.463 \\
2.906\end{array}$ & $\begin{array}{r}10.239 \\
4.715 \\
3.468 \\
2.907\end{array}$ & $\begin{array}{l}9.664 \\
4.646 \\
3.442 \\
2.895\end{array}$ & $\begin{array}{r}33.065 \\
8.126 \\
5.109 \\
3.973\end{array}$ & $\begin{array}{r}16.14 \\
6.476 \\
4.479 \\
3.625\end{array}$ & $\begin{array}{r}16.577 \\
6.501 \\
4.487 \\
3.627\end{array}$ & $\begin{array}{r}19.972 \\
7.156 \\
4.780 \\
3.802\end{array}$ \\
\hline
\end{tabular}

Let $\sigma_{1}^{2}$ and $\sigma_{2}^{2}$ denote the variances of the two normal populations and let $s_{1}^{2}$ and $s_{2}^{2}$ be their inde-

TABLE 6. Comparison of the tabulated and calculated values of $\lambda(\alpha, \beta, n)$ 
pendent sample estimates based on $n_{1}$ and $n_{2}$ d.o.f. respectively. Our hypotheses are $H_{1}: \sigma_{1}^{2}=\sigma_{2}^{2}$ and $H_{2}: \sigma_{1}^{2}>\sigma_{2}^{2}$. The statistic $F=s_{1}^{2} / s_{2}^{2}$ is used to accept or reject $H_{1}$. For a given level of significance $\alpha$, if $F \leq F_{\alpha}$ we accept $H_{1}$ and accept $H_{2}$ when $F>F_{\alpha}$. Let $\bar{\phi}$ denote the true value of $\sigma_{1}^{2} / \sigma_{2}^{2}$. It has been shown in [2] and [4] that the probability of an error of the second kind will be exactly $\beta$ if

$$
\phi\left(\alpha, \beta, n_{1}, n_{2}\right)=\frac{F_{\alpha}\left(n_{1}, n_{2}\right)}{F_{1-\beta}\left(n_{1}, n_{2}\right)}=F_{\alpha}\left(n_{1}, n_{2}\right) F_{\beta}\left(n_{2}, n_{1}\right) .
$$

The operating characteristics $(\phi, \beta)$ for one-sided alternatives have been given in [2] and [4]. We shall develop here certain approximate formulas for $\phi$ in terms of $\alpha, \beta, n_{1}$, and $n_{2}$.

By taking one-half the logarithm of (55) we obtain

$$
\frac{1}{2} \log \phi=z_{\alpha}\left(n_{1}, n_{2}\right)+z_{\beta}\left(n_{2}, n_{1}\right)
$$

where $z_{\alpha}$ denotes the $\alpha$-probability point of Fisher's $z$-distribution. For purposes of approximation, (56) has decided advantage over (55) in that it enables us to make better use of the Cornish-Fisher approximation [13] since the z-distribution approaches normality relatively faster than the $F$-distribution. We shall employ the following approximations [14] for the cumulants of $z$ :

(i) $\kappa_{1}=\frac{1}{2}\left(\frac{1}{n_{2}}-\frac{1}{n_{1}}\right)$

(ii) $\kappa_{2}=\frac{1}{2}\left(\frac{1}{n_{1}}+\frac{1}{n_{2}}\right)$

(iii) $\kappa_{s}=\frac{(s-2) !}{2}\left(\frac{1}{\left(n_{2}-1\right)^{s-1}}+\frac{(-1)^{s}}{\left(n_{1}-1\right)^{s-1}}\right)(s>1)$.

Let $z_{1}$ and $z_{2}$ denote the mathematical expectation of $z$ under $H_{1}$ and $H_{2}$, respectively. Consequently we have

$$
\begin{aligned}
z_{1} & \simeq \frac{1}{2}\left(\frac{1}{n_{2}}-\frac{1}{n_{1}}\right) \\
z_{2} & \simeq \frac{1}{2}\left(\frac{1}{n_{2}}-\frac{1}{n_{1}}\right)+\frac{1}{2} \log \phi \\
V(z) & \simeq \frac{1}{2}\left(\frac{1}{n_{1}}+\frac{1}{n_{2}}\right)
\end{aligned}
$$

By applying the results of section 3 to $z$, it is found that

$$
2\left(\frac{1}{n_{1}}+\frac{1}{n_{2}}\right)=\left(\frac{\log \phi}{K_{\alpha}+K_{\beta}}\right)^{2}
$$

which may be rewritten as

$$
\log \phi=\frac{2\left(K_{\alpha}+K_{\beta}\right)}{\sqrt{h}},
$$

where $h=\frac{2 n_{1} n_{2}}{n_{1}+n_{2}}$. By using $\kappa_{2}=\left(\frac{1}{n_{2}-1}+\frac{1}{n_{1}-1}\right)$

there results

$$
2\left(\frac{1}{n_{1}-1}+\frac{1}{n_{2}-1}\right)=\left(\frac{\log \phi}{K_{\alpha}+K_{\beta}}\right)^{2}
$$

Directly applying to (56) the Cornish-Fisher approximation $\lceil 13\rceil$ for the percentage points of the $z$-distribution in conjunction with Cochran's $\lambda$-corrections [15], we obtain (58) and the following two expressions for $\log \phi$ :

$$
\log \phi=2\left(\frac{K_{\alpha}}{\sqrt{h-\lambda_{\alpha}}}+\frac{K_{\beta}}{\sqrt{h-\lambda_{\beta}}}\right)
$$

$\log \phi=2\left(\frac{K_{\alpha}}{\sqrt{h-\lambda_{\alpha}}}+\frac{K_{\beta}}{\sqrt{h-\lambda_{\beta}}}\right)+\frac{1}{8}\left(\frac{1}{n_{2}}-\frac{1}{n_{1}}\right)^{\bullet}\left(K_{\alpha}^{2}-K_{\beta}^{2}\right)$

\begin{tabular}{|c|c|c|c|c|c|c|}
\hline \multicolumn{7}{|c|}{$\alpha=.05, \beta=.05$} \\
\hline & 5 & 10 & 15 & 20 & 30 & 60 \\
\hline \multirow{2}{*}{5} & $\int 26.312$ & 15. 674 & 13.082 & 11. 918 & 10.844 & 9.839 \\
\hline & 25.51 & 15.75 & 13.40 & 12.36 & 11.39 & 10.49 \\
\hline \multirow{2}{*}{10} & 15. 674 & 8.910 & 7. 240 & 6.486 & 5.787 & 5. 130 \\
\hline & 15. 75 & 8.870 & 7.237 & 6.513 & 5.844 & 5. 223 \\
\hline \multirow{2}{*}{15} & 13. 082 & 7. 240 & 5. 787 & 5. 130 & 4. 516 & 3.937 \\
\hline & 13.40 & 7. 237 & 5. 777 & 5. 128 & 4.527 & 3.967 \\
\hline \multirow{2}{*}{20} & $\int 11.918$ & 6.486 & 5. 130 & 4. 516 & 3. 937 & 3.390 \\
\hline & $\left\{\begin{array}{l}12.36 \\
12.3\end{array}\right.$ & 6.513 & 5. 128 & 4. 512 & 3.939 & 3.402 \\
\hline \multirow{2}{*}{30} & f 10.844 & 5. 787 & 4. 516 & 3. 937 & 3. 390 & 2. 866 \\
\hline & 11.39 & 5.844 & 4.527 & 3. 939 & 3. 389 & 2. 869 \\
\hline \multirow{2}{*}{60} & \{ 9.839 & 5. 130 & 3.937 & 3.390 & 2. 866 & 2. 355 \\
\hline & 10.49 & 5.223 & 3.967 & 3.402 & 2.869 & 2.354 \\
\hline
\end{tabular}

where $\lambda_{\epsilon}$ is given by

$\begin{array}{llllll}\epsilon & .75 & .50 & .25 & .05 & .01 \\ \lambda_{\epsilon} & .5758 & .5000 & .5758 & .9509 & 1.4020\end{array}$

Formulas (58), (59), and (60) are not changed when

\begin{tabular}{|c|c|c|c|c|c|c|}
\hline \multicolumn{7}{|c|}{$a=.01, \beta=.01$} \\
\hline$n_{2}$ & 5 & 10 & 15 & 20 & 30 & 60 \\
\hline \multirow{2}{*}{5} & $\int 135.047$ & 57.720 & 43. 294 & 37.428 & 32.304 & 27.816 \\
\hline & 120.3 & 56.65 & 44. 29 & 39.19 & 34.69 & 30.72 \\
\hline \multirow{2}{*}{10} & 57.720 & 23.893 & 17. 434 & 14. 791 & 12. 474 & 10.436 \\
\hline & 56.65 & 23.51 & 17.35 & 14. 84 & 12.65 & 10.74 \\
\hline \multirow{2}{*}{15} & 43. 294 & 17.434 & 12.474 & 10.436 & 8. 650 & 7. 082 \\
\hline & 44.29 & 17.34 & 12.41 & 10.41 & 8. 679 & 7.168 \\
\hline \multirow{2}{*}{20} & 37.428 & 14. 791 & 10.436 & 8. 650 & 7. 082 & 5.697 \\
\hline & 39.19 & 14.84 & 10.41 & 8.630 & 7. 082 & 5. 731 \\
\hline \multirow{2}{*}{30} & 32.304 & 12. 474 & 8. 650 & 7. 082 & 5. 697 & 4. 471 \\
\hline & $\left\{\begin{array}{l}34.69 \\
\text {. }\end{array}\right.$ & 12.65 & 8.679 & 7. 682 & 5. 693 & 4. 479 \\
\hline \multirow{2}{*}{60} & $\int 27.816$ & 10.436 & 7. 082 & 5. 697 & 4. 471 & 3.372 \\
\hline & $\left\{\begin{array}{l}30.72\end{array}\right.$ & 10. 74 & 7.168 & 5. 731 & 4. 479 & 3.372 \\
\hline
\end{tabular}
$n_{1}$ and $n_{2}$ are interchanged. Formulas (60) and (61) are identical when either $\alpha=\beta$ or $n_{1}=n_{2}$, or both.

TABLE 7A. ${ }^{\mathrm{a}}$ Comparison of the calculated (formula 61) and tabulated values of $\varphi\left(\alpha, \beta, n_{1}, n_{2}\right)$

a Figures in bold face type are taken from [4].

TABLE 7B, ${ }^{\mathrm{a}}$ Comparison of calculated (formula 61) and tabulated values of $\varphi\left(\alpha, \beta, n_{1}, n_{2}\right)$

a Figures in bold face type are taken from [4]. 
TABLE 7C. Comparison of calculated (formula 61) and tabulated values of $\varphi\left(\alpha, \beta, n_{1}, n_{2}\right)$

\begin{tabular}{|c|c|c|c|c|c|c|c|}
\hline \multicolumn{8}{|c|}{$a=.05, \beta=.25$} \\
\hline$n_{2}$ & & 5 & 10 & 15 & 20 & 30 & 60 \\
\hline \multirow[b]{2}{*}{5} & & 9. 742 & 7. 371 & 6. 674 & 6. 338 & 6.012 & 5. 693 \\
\hline & & 9. 569 & 7.507 & 6. 900 & 6.609 & 6. 327 & 6.052 \\
\hline \multirow{2}{*}{10} & i & 6.345 & 4. 632 & 4. 112 & 3.837 & 3.607 & 3. 359 \\
\hline & \{ & 6.285 & 4. 620 & 4. 123 & 3.882 & 3.646 & 3.614 \\
\hline \multirow{2}{*}{15} & j & 5. 464 & 3. 192 & 3. 432 & 3.195 & 2. 959 & 2. 722 \\
\hline & \{ & 5. 469 & 3.902 & 3.428 & 3.197 & 2.969 & 2.741 \\
\hline \multirow{2}{*}{20} & j & 5.061 & 3.578 & 3. 116 & 2.886 & 2. 655 & 2. 421 \\
\hline & \{ & 5. 102 & 3.577 & 3. 113 & 2.885 & 2. 658 & 2. 429 \\
\hline \multirow{2}{*}{30} & j & 4. 682 & 3. 264 & 2.815 & 2.590 & 2. 361 & 2.125 \\
\hline & \{ & 4. 759 & 3. 273 & 2. 815 & 2. 589 & 2. 361 & 2. 127 \\
\hline \multirow{2}{*}{60} & j & 4. 325 & 2. 964 & 2. 526 & 2. 303 & 2. 072 & 1. 828 \\
\hline & \{ & 4. 439 & 2.987 & 2.533 & 2.306 & 2.073 & 1.828 \\
\hline
\end{tabular}

TABLE 8. Values of $\varphi\left(\alpha, \beta, n_{1}, n_{2}\right)$ from $\log \varphi=$ $\left(K_{\alpha}+K_{\beta}\right) \sqrt{2\left(\frac{1}{n_{1}-1}+\frac{1}{n_{2}-1}\right)}$

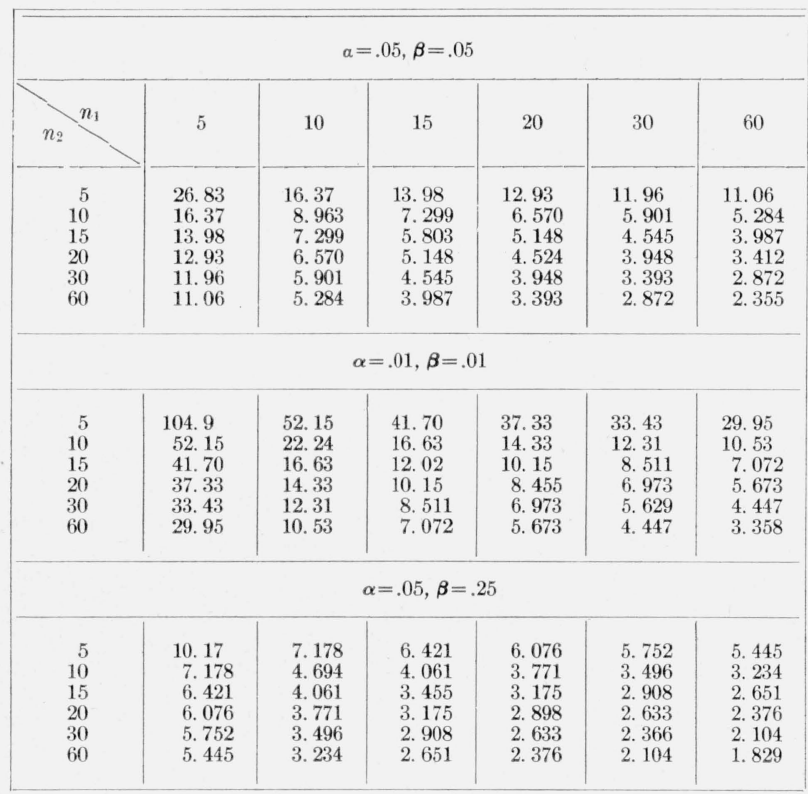

It appears on the basis of several computations (not given here) that formula (58) is likely to give values of $\phi$ that are much lower than its tabulated values [4], and consequently the sample sizes given by it will fall below the minimum desired. For $\alpha=\beta$ formula (61), then equivalent to (60), gives values of $\phi$ which are much closer to its tabulated values (table 7). This is also true of (61) for $\beta>\alpha$. If in addition to $\alpha=\beta, n_{1}=n_{2}=n$, then from (60)

$$
n=\lambda \alpha+\Psi\left(\frac{K_{\alpha}+K_{\beta}}{\log \phi}\right)^{2}
$$

We have not found any formula which will give an approximately correct answer for degrees of freedom as small as, say, $n_{1}=n_{2}=5$. The question of finding appropriate sample sizes for $n_{1} \neq n_{2}$ cannot ordinarily be answered without the help of tables.
In such a situation, however, the experimentalist has no choice in the determination of $n_{1}$ and $n_{2}$. If it is decided in advance to maintain a certain ratio between $n_{1}$ and $n_{2}$ (this appears to be more often the case in practice) a formula would be more practical to use than the existing tables. Since formula (61) seems to be very complicated to use, we recommend the use of (59). Values of $\phi$ as given by this formula are given in table 8 . It appears that for $\alpha=\beta=.05$, formula (59) will always give sufficient sample sizes; but for $\alpha=\beta=.01$, it will give values slightly less than actually needed.

This paper could not have been written without the constant encouragement of Churchill Eisenhart. The author acknowledges with pleasure the help given by Lola S. Deming and Celia S. Martin for doing the enormous computations. Thanks are also due to Elizabeth Shuhany of the Statistical Laboratory, Boston University, for some computational help.

\section{References}

[1] J. Neyman and B. Tokarska, Errors of the second kind in testing "Student's" hypotheses, J. Am. Stat. Assoc. 31, 318 (1936).

[2] Charles D. Ferris, Frank E. Grubbs, and Chalmers L. Weaver, Operating characteristics for the common statistical tests of significance, Ann. Math. Stat. 17, 178 (1946).

[3] J. H. Curtiss, Acceptance sampling by variables, with special reference to the case in which quality is measured by average or dispersion, J. Research, NBS 39, 271 (1947) RP 1827.

[4] Churchill Eisenhart, Millard W. Hastay, and W. Allen Wallis, Selected techniques of statistical analysis (McGraw-Hill Book Co., Inc., New York, N. Y., 1947).

[5] J. Neyman, On the problem of most efficient tests of statistical hypotheses, Phil. Trans. Roy. Soc. 231, $289(1932-3)$.

[6] "Student" (W. S. Gossett), The probable error of a mean, Biometrika 6, 1 (1908).

[7] K. Pearson, Tables for statisticians and biometricians, Part II (University of Cambridge, England, 1931).

[8] P. C. Tang, The power function of the analysis of variance tests with tables and illustrations of their use, Statistical Research Memoirs, Vol. 2 (University of London, 1938).

[9] E. Lehmer, Inverse tables of probability of errors of the "second kind", Ann. Math. Stat. 15, 388 (1944).

[10] Uttam Chand, Distributions related to the comparison of two means and two regression coefficients, Ann. Math. Stat. 21, 507 (1950).

[11] M. S. Bartlett and D. G. Kendall, The statistical analysis of variance heterogeneity and the logarithmic transformation, Supp. J. Roy. Stat. Soc. 8, 128 (1946).

[12] E. T. Whittaker and G. M. Watson, A course of modern analysis, 4th ed. (Cambridge University Press, 1935).

[13] E. A. Cornish and R. A. Fisher, Moments and cumulants in the specification of distributions, Rev. Inst. Intl. Stat. 14, 1 (1937)

[14] J. Wishart, The cumulants of the $z$ and of the logarithmic $\chi^{2}$ and $t$ distributions, Biometrika 34, 170 (1947).

[15] W. G. Cochran, Note on an approximate formula for significance levels of $z$, Ann. Math. Stat. 11, 93 (1940).

[16] R. A. Fisher, On the dominance ratio, Proc. Roy. Soc. Edinburgh, 42, 321 (1921-22). See also [4] chapter 16.

[17] M. S. Bartlett, Square root transformation in analysis of variance, Suppl. J. Roy. Stat. Soc. 3, 68 (1936). See also [4] chapter 16.

[18] R. A. Fisher, Statistical methods for research workers (Oliver and Boyd, Edinburgh). 


\section{Appendix. Tabular summary of single-sampling formulas for testing hypotheses}

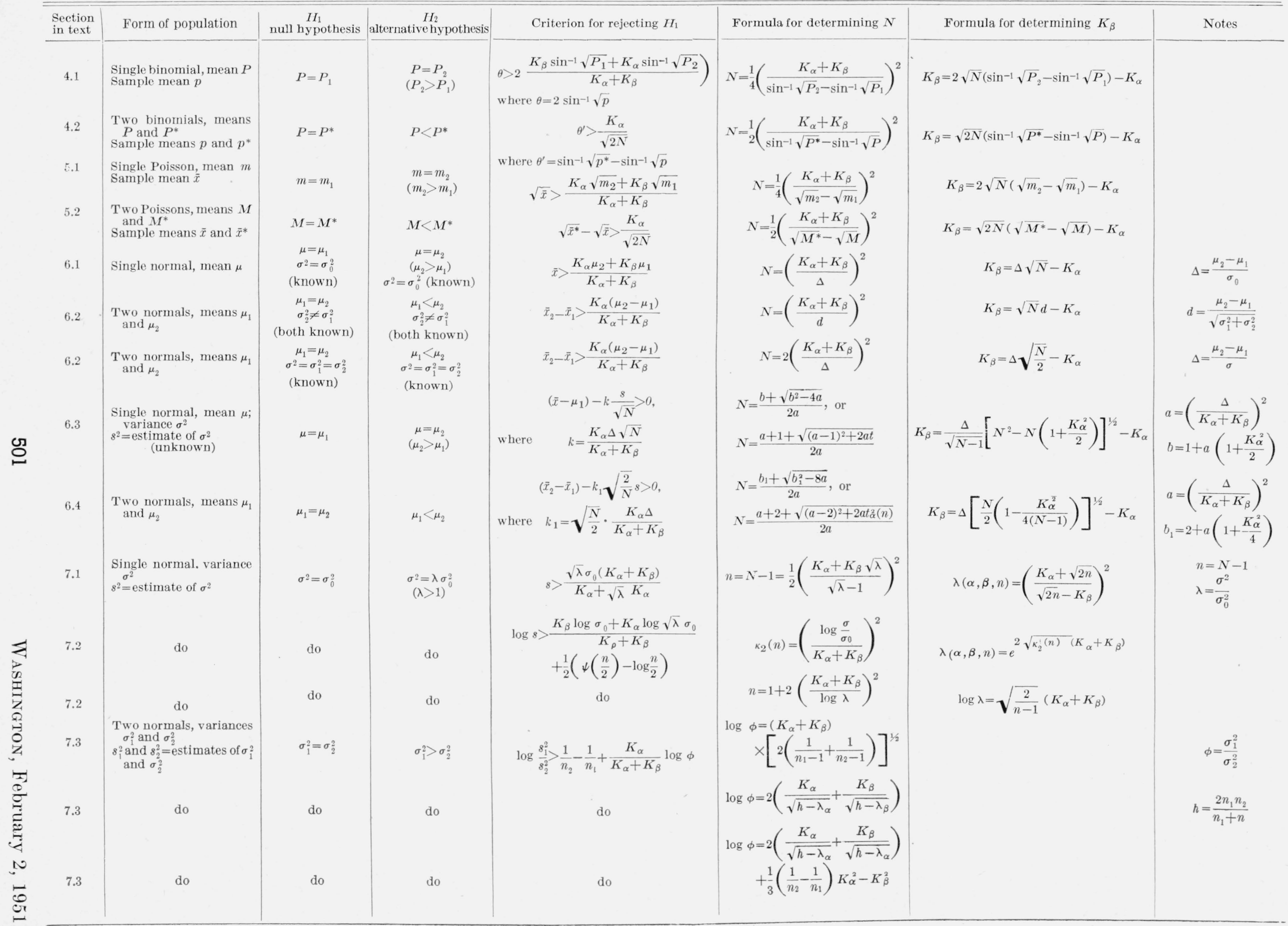

